\title{
Local Unitary Classification of Arbitrary Dimensional Multipartite Pure States
}

\author{
Bin Liu ${ }^{1}$, Jun-Li $\mathrm{Li}^{1}$, Xikun $\mathrm{Li}^{1}$, and Cong-Feng Qiao ${ }^{1,2 *}$ \\ ${ }^{1}$ Department of Physics, Graduate University of Chinese Academy of Sciences \\ YuQuan Road 19A, Beijing 100049, China \\ ${ }^{2}$ Theoretical Physics Center for Science Facilities (TPCSF), CAS \\ YuQuan Road 19B, Beijing 100049, China
}

\begin{abstract}
We propose a practical entanglement classification scheme for general multipartite pure states in arbitrary dimensions under local unitary equivalence by exploiting the high order singular value decomposition technique and local symmetries of the states. By virtue of this scheme, the method of determining the local unitary equivalence of $n$-qubit states proposed by Kraus is extended to the case for arbitrary dimensional multipartite states.
\end{abstract}

PACS numbers: 03.67.Mn, 02.10.Xm, 03.65.Ud

Entanglement is one of the most extraordinary features of quantum theory. It lies at the heart of quantum information theory and is now regarded as a key physical resource in realizing many quantum information tasks, such as teleportation and quantum computation, etc. [1. In practice, people may confront various forms of entangled states even in one single physical system. Though superficially showing up with different features, usually not all these entangled states are functionally independent; they may be intrinsically the same where the entanglement property is concerned. Two entangled states are said to be equivalent in implementing the same quantum information task if they can be obtained with certainty

\footnotetext{
${ }^{*}$ Corresponding author.
} 
from each other via local operation and classical communication (LOCC). Theoretically, this LOCC equivalent class is defined such that within this class any two quantum states are interconvertible by local unitary (LU) operators [2]. If we release the constraint of the local unitary operator to invertible local transformation, the LOCC equivalence then turns to the widely discussed stochastic local operation and classical communication (SLOCC) equivalence.

The classification of a bipartite system under LU can be done by singular value (Schmidt) decomposition. For the classification of a pure multipartite entangled state, a canonical method was proposed in Ref.[3], though it was given only in a set of constraints on the coefficients of quantum state. Later, this method was reformulated into a compact form [4], where the existence of SLOCC normal forms was also proved. Recently, by introducing the standard form for multipartite states, Kraus proposed a general way to determine the local unitary transformation between two LU equivalent $n$-qubit states [5]. Even though these beautiful results have been obtained, a generic method or methodology is still missing in the classification of general multipartite entangled states [6].

Much research indicates that symmetry study is very helpful in the classification of a general multipartite system. It is broadly realized that making restrictions on the quantum states, i.e., satisfying some symmetric properties, is a feasible way in the entanglement classification [7, 8]. By virtue of the Majorana representation, a lot of effort was spend on the study of the relation between permutation symmetry and the classification of a multipartite entangled state under both LOCC and SLOCC [9, 10, 11]. It was recently realized that there exists one kind of local (internal) symmetry under SLOCC equivalence for a continuously entangled state [12]. Unlike the permutation symmetry among different partites, the internal symmetry is of a localized one. In Refs. [13, 14, 15], people investigated the nature of the $n$-qubit entangled state via analyzing the stabilizers of the symmetric state, which can also be regarded as a local unitary transformation.

In this work, we present a new and easy to operate entanglement classification method under the LU equivalence, by virtue of the technique of high order singular decomposition (HOSVD) [16, 17] and by exploiting the local symmetries of the state [12]. The procedure of this method goes as follows: Express the quantum state into a complex tensor, analyze 
the possible local symmetry property of the quantum state, apply the HOSVD on the tensor form of the quantum state, and then fully classify a general multipartite state into LU inequivalent classes by sequential use of HOSVD and local unitary symmetry.

A general $N$-partite entangled quantum state in dimension $I_{1} \times I_{2} \times \cdots \times I_{N}$ can be formulated as the following form:

$$
|\Psi\rangle=\sum_{i_{1}=1, i_{2}=1, \ldots, i_{N}=1}^{I_{1}, I_{2}, \cdots, I_{N}} \psi_{i_{1} i_{2} \ldots i_{N}}\left|i_{1}\right\rangle\left|i_{2}\right\rangle \ldots\left|i_{N}\right\rangle
$$

where $\psi_{i_{1} i_{2} \ldots i_{N}} \in \mathbb{C}$ are coefficients of the quantum state in representative bases. Two quantum states are said to be equivalent if they are interconvertible by a certain type of operators, which can be schematically expressed as

$$
\begin{aligned}
\left|\Psi^{\prime}\right\rangle & =\bigotimes_{i}^{N} U^{(i)}|\Psi\rangle \\
& =\sum_{\substack{i_{1}, i_{2}, \cdots, i_{N} \\
i_{1}^{\prime}, i_{2}^{\prime}, \cdots, i_{N}^{\prime}}} \psi_{i_{1} i_{2} \cdots i_{N}} u_{i_{1}^{\prime} i_{1}}^{(1)}\left|i_{1}^{\prime}\right\rangle u_{i_{2}^{\prime} i_{2}}^{(2)}\left|i_{2}^{\prime}\right\rangle \cdots u_{i_{N}^{\prime} i_{N}}^{(N)}\left|i_{N}^{\prime}\right\rangle \\
& =\sum_{i_{1}, i_{2}, \cdots, i_{N}} \psi_{i_{1} i_{2} \cdots i_{N}}^{\prime}\left|i_{1}, i_{2}, \cdots, i_{N}\right\rangle
\end{aligned}
$$

Here, the coefficients $\psi_{i_{1} i_{2} \ldots i_{N}}$ can also be treated as the entries of a tensor $\Psi$, and hence the quantum states can be represented by high dimensional complex tensors. From the tensor form of $\Psi$, the operator $U^{(n)}$ acting on the $n$th partite is defined as

$$
\left(U^{(n)} \Psi\right)_{i_{1} i_{2} \cdots i_{n-1} i_{n}^{\prime} i_{n+1 \cdots i_{N}}} \equiv \sum_{i_{n}} \psi_{i_{1} i_{2} \cdots i_{n-1} i_{n} i_{n+1} \cdots i_{N}} u_{i_{n}^{\prime} i_{n}}^{(n)}
$$

In case $U^{(n)}$ 's are unitary operators, quantum state $\Psi^{\prime}$ is said to be $L U$ equivalent to $\Psi$, i.e.,

$$
\Psi^{\prime}=\bigotimes_{i} U^{(i)} \Psi
$$

Suppose a concerned quantum state is inherited with local symmetries, like

$$
\bigotimes_{n} P^{(n)} \Psi=\Psi
$$


and is always assisted with classical communication hereafter in our discussion; the local symmetry group $G=\left\{g \mid g=\bigotimes_{n} P^{(n)}\right\}$ then forms the stabilizer of $\Psi$. Here $P^{(n)}$ is the operator that acts on the $n$th partite. From Eq.(44), we can get

$$
\begin{aligned}
\Psi^{\prime}= & U^{(1)} \otimes U^{(2)} \otimes \cdots \otimes U^{(i)} \otimes \cdots \otimes U^{(N)} \Psi \\
= & U^{(1)} P^{(1)} \otimes U^{(2)} P^{(2)} \otimes \cdots \\
& \otimes U^{(i)} P^{(i)} \otimes \cdots \otimes U^{(N)} P^{(N)} \Psi \\
= & \bigotimes_{i} U^{(i)} P^{(i)} U^{(i) \dagger} \Psi^{\prime}
\end{aligned}
$$

with $U^{(i)}$ the unitary operators. Clearly, every local unitary equivalent state $\Psi^{\prime}$ has the local symmetry of $\left(\bigotimes_{i} U^{(i)}\right) \cdot\left(\bigotimes_{j} P^{(j)}\right) \cdot\left(\bigotimes_{k} U^{(k) \dagger}\right)$, which is isomorphic to that of $\Psi$. Consequently, we have the following proposition.

Proposition 1 If $\Psi^{\prime}$ is local unitary equivalent to $\Psi$, then the stabilizers of the local symmetries of the two states are unitarily equivalent.

By taking $|\psi\rangle=\alpha|11\rangle+\beta|22\rangle+\gamma|33\rangle+\gamma|44\rangle$, where $|\alpha|^{2}+|\beta|^{2}+2|\gamma|^{2}=1$ as an example, this $4 \times 4$ entangled state can be expressed in a matrix form:

$$
\Psi_{4 \times 4}=\left(\begin{array}{cccc}
\alpha & 0 & 0 & 0 \\
0 & \beta & 0 & 0 \\
0 & 0 & \gamma & 0 \\
0 & 0 & 0 & \gamma
\end{array}\right)
$$

It is invariant under the local transformation $U^{(1)}=\left\{e^{i \theta_{1}}, e^{i \theta_{2}}, u\right\}$ and $U^{(2)}=\left\{e^{-i \theta_{1}}, e^{-i \theta_{2}}, u^{*}\right\}$, i.e.,

$$
U^{(1)} \cdot \Psi \cdot\left(U^{(2)}\right)^{\mathrm{T}}=\Psi
$$

The $\Psi_{4 \times 4}$ is then a $U^{(1)} \otimes U^{(2)}$ invariant quantum state.

To study the LU classification of a multipartite system, it is convenient for us to use the notation of Ref.[16] and to define the matrix unfolding of the tensor $\Psi \in \mathbb{C}^{I_{1} I_{2} \cdots I_{N}}$ with the $n$th index as

$$
\Psi_{(n)} \in \mathbb{C}^{I_{n} \times\left(I_{n+1} I_{n+2} \cdots I_{N} I_{1} I_{2} \cdots I_{n-1}\right)} .
$$


Here $\Psi_{(n)}$ is an $I_{n} \times\left(I_{n+1} I_{n+2} \cdots I_{N} I_{1} I_{2} \cdots I_{n-1}\right)$ matrix. Considering the high order singular value decomposition developed in Ref. [16], there exists a core tensor $\Sigma$ for each $\Psi$, that is,

$$
\Psi=U^{(1)} \otimes U^{(2)} \cdots \otimes U^{(N)} \Sigma
$$

where $\Sigma$ forms a same order tensor in the Hilbert space $I_{1} \times I_{2} \times \cdots \times I_{N}$. Any $N$-1 order tensor $\Sigma_{i_{n}=i}$, obtained by fixing the $n$th index to $i$, has the following properties [16]:

$$
\left\langle\Sigma_{i_{n}=i}, \Sigma_{i_{n}=j}\right\rangle=\delta_{i j} \sigma_{i}^{(n) 2}
$$

with $\sigma_{i}^{(n)} \geq \sigma_{j}^{(n)}$ and $\forall i<j$ for all possible values of $n$. Here, the singular value $\sigma_{i}^{(n)}$ symbolizes the Frobenius norm $\sigma_{i}^{(n)}=\left\|\Sigma_{i_{n}=i}\right\| \equiv \sqrt{\left\langle\sum_{i_{n}=i}, \Sigma_{i_{n}=i}\right\rangle}$, where the inner product $\langle\mathcal{A}, \mathcal{B}\rangle \equiv \sum_{i_{1}} \sum_{i_{2}} \cdots \sum_{i_{N}} b_{i_{1} i_{2} \ldots i_{N}} a_{i_{1} i_{2} \ldots i_{N}}^{*}$.

From the definition, we know that if the quantum state $\Psi^{\prime}$ is LU equivalent to $\Psi$, then they can be transformed into the same core tensor $\Sigma$. However, because there is no information on whether different core tensors are local unitary equivalent or not, $\Sigma$ has nothing to do with the entanglement classification of the multipartite state yet. Hence, to achieve the entanglement classification from this point, the following analysis is necessary.

Since every unitarily equivalent quantum state possesses the same local symmetric property, we can pick up a typical state to represent one equivalent class. A natural choice is the core tensor for each class. In the matrix unfolding form of the core tensor, $\Sigma_{(n)}$ can be expressed as

$$
\Psi_{(n)}=U^{(n)} \cdot \Sigma_{(n)} \cdot\left(U^{(n+1)} \otimes \cdots \otimes U^{(N)} \cdot U^{(1)} \cdots U^{(n-1)}\right)^{\mathrm{T}}
$$

where $U^{(n)}$ is constructed by the left singular vectors of $\Psi_{(n)}$.

There exist such quantum states $\Psi$ that some of the singular values of their unfolding matrix $\Psi_{(n)}$ are identical, which is similar to the singular value $\gamma$ in the matrix case in Eq.(7) and is also stated in Property 4 of Ref.[16]. The $\Psi_{(n)}$ has the following property:

$$
\Psi_{(n)}=U^{(n)} P^{(n)} \cdot \Sigma_{(n)}^{\prime} \cdot\left(U^{(n+1)} \otimes \cdots \otimes U^{(N)} \otimes U^{(1)} \cdots U^{(n-1)}\right)^{\mathrm{T}}
$$

where $P^{(n)}$ is a block-diagonal matrix consisting of unitary blocks with the same partitions 
as that of the identical singular values, and $\Sigma_{(n)}^{\prime}=P^{(n) \dagger} \Sigma_{(n)}$. For such states we have

$$
\Sigma^{\prime}=\bigotimes_{n=1}^{N} P^{(n) \dagger} \Sigma
$$

Here, $P^{(n)}=\operatorname{diag}\left\{e^{i \theta_{1}}, \cdots, e^{i \theta_{I_{n}}}\right\}$ in case there is no identical singular value for $\Sigma_{(n)}$. Thus, if $\Sigma$ is a HOSVD of $\Psi$, then the $\Sigma^{\prime}$ is as well. In other words, the HOSVD of $\Psi$ possesses local symmetry properties of $\bigotimes_{n=1}^{N} P^{(n) \dagger}$.

From the above discussion, we may conclude that if we introduce the symmetry property to compensate the nonuniqueness of the core tensor, it will then serve as the unique representative state for each local unitary equivalent class. We call the representative state $\Sigma$ associated with specific symmetry group $\bigotimes_{n=1}^{N} P^{(n)}$ the unique canonical form of the entanglement class under LU equivalence. Two quantum states are LU equivalent if and only if they have the same core tensor (representative state) or their core tensors are related by the symmetry group $\bigotimes_{n=1}^{N} P^{(n)}$.

Proposition 2 The high order singular value decomposition of multipartite state is a $L U$ classification of entanglement up to the local symmetry $\bigotimes_{n=1}^{N} P^{(n)}$.

Proof: First, it is easy to see that if two quantum state are LU equivalent, i.e., $\Psi^{\prime}=\otimes_{i}^{N} U^{(i)} \Psi$, then they can be transformed into the same core tensor $\Sigma$. Second, suppose two core tensors are related by local unitary transformations $\Sigma^{\prime}=\bigotimes_{i} V^{(n)} \Sigma$, where $\bigotimes_{i} V^{(n)}$ are different from $\bigotimes_{n=1}^{N} P^{(n)}$; then by taking the $n$th unfolding as an example, we have

$$
\Psi_{(n)}^{\prime}=U^{(n)} \cdot \Sigma_{(n)}^{\prime} \cdot(\cdots)=U^{(n)} V^{(n)} \cdot \Sigma_{(n)} \cdot(\cdots)
$$

This means that the singular values are not uniquely determined, which is in contradiction with Property 4 of Ref. [16]. Therefore, we can conclude that the core tensor $\Sigma$ up to the local symmetry $\bigotimes_{n=1}^{N} P^{(n)}$ is unique, or, in other words, the core tensor of the quantum state can be treated as the LU classification of a multipartite quantum state up to the local symmetry $\bigotimes_{n=1}^{N} P^{(n)}$. QED.

Now, for a general arbitrary dimensional $N$-partite pure state, the canonical form can be constructed in the following way. First, express the tensor form quantum state in matrix 
unfolding form $\Psi_{(n)}$ [see Eq.(9) ]. Then, compute the singular value decomposition of the matrix $\Psi_{(n)}=U^{(n)} \Lambda V^{(n)}$. Here $N$ unitary matrices $U^{(n)}$, composed by left singular vectors of $\Psi_{(n)}$, for $N$ indices will be obtained, and this is a simple process while only matrix operations are involved. Finally, the core tensor is constructed by

$$
\Sigma=\bigotimes_{n=1}^{N} U^{(n) \dagger} \Psi
$$

The representative state $\Sigma$ with its associated symmetry group in the form of Eq. (14) will serve as the unique canonical form of $\Psi$.

Given two canonical forms, i.e., the obtained representative states $\Sigma$ and $\widetilde{\Sigma}$ with some local symmetries of $\bigotimes_{n} Q^{(n)}$ and $\bigotimes_{n} \widetilde{Q}^{(n)}$, respectively, the procedure of verifying the existence of a local symmetry that connects the two representative states is straightforward. If singular values of these two representative states or the sizes of the unitary blocks of local symmetries are not the same, obviously there does not exist such a local symmetry that the two tensors can be transformed into each other. Otherwise, the existence of the local symmetry is equivalent to the existence of solutions to the equation $\widetilde{\Sigma}=\bigotimes_{n} Q^{(n)} \Sigma$ (or $\Sigma=\bigotimes_{n} \widetilde{Q}^{(n)} \widetilde{\Sigma}$ ), where the elements in $\Sigma$ and $\widetilde{\Sigma}$ are known quantities whereas they are unknown variables in $Q^{(n)}\left(\widetilde{Q}^{(n)}\right)$. By virtue of the mathematical technique relinearization [18, 19], one can readily know whether the solutions to the equations exist or not (practical examples are given in Ref. [20]).

Last, we show briefly the relation of the core tensor and Kraus's canonical form $\left|\Psi_{\text {st }}\right\rangle$ in Ref. [5]. The sorted trace decomposition of the $n$th partite requires $\operatorname{Tr}_{\neg i_{n}}\left(\left|\Psi_{\text {st }}\right\rangle\left\langle\Psi_{\text {st }}\right|\right)$ to be diagonalized. For a general quantum state

$$
\begin{aligned}
\left|\Psi_{\text {st }}\right\rangle & =\sum_{i_{1}, i_{2}, \cdots, i_{N}}^{I_{1}, I_{2}, \cdots I_{N}} \psi_{i_{1} i_{2} \cdots i_{N}}\left|i_{1}, i_{2}, \cdots, i_{N}\right\rangle \\
& =\sum_{i_{n}}\left|i_{n}\right\rangle \sum_{\neg i_{n}} \psi_{i_{1} i_{2} \cdots i_{n-1} i_{n} i_{n+1} \cdots i_{N}}\left|i_{1} i_{2} \cdots i_{n-1} i_{n+1} \cdots i_{N}\right\rangle,
\end{aligned}
$$

the requirement of trace diagonalization asks

$$
\begin{array}{r}
\sum_{\neg j} \psi_{i_{1} i_{2} \cdots i_{n-1} j i_{n+1} \cdots i_{N}}^{*} \psi_{i_{1} i_{2} \cdots i_{n-1} j^{\prime} i_{n+1} \cdots i_{N}}=\lambda_{j}^{(n)} \delta_{j j^{\prime}}, \\
\lambda_{1}^{(n)} \geq \lambda_{2}^{(n)} \geq \cdots \geq \lambda_{I_{n}}^{(n)} \geq 0 .
\end{array}
$$


Here, $\lambda_{j}^{(n)}=\sum_{\neg j} a_{i_{1} i_{2} \cdots i_{n-1} j i_{n+1} \cdots i_{N}}^{*} a_{i_{1} i_{2} \cdots i_{n-1} j i_{n+1} \cdots i_{N}}$ is just the Frobenius norm $\left\|\left(\Psi_{\text {st }}\right)_{i_{n}=j}\right\|^{2}$ in high order singular decomposition employed in our classification approach.

In summary, by virtue of the symmetry property study and tensor decomposition we propose an effective and easy to operate method for the local unitary classification of a multipartite entangled state, which splits the mathematically difficult problem into different relatively easier ones. That is, we first play tensor decomposition on the quantum state and obtain the quasinormal form (core tensor), where many different quasinormal forms may actually belong to the same entanglement class; then, by exploiting the local symmetries between the quasinormal forms, we arrive at a uniquely defined entanglement class. By this approach, the verification of LU equivalence of $n$-qubit states proposed by Kraus is extended to the case for arbitrary dimensional multipartite states in a more easily understandable and computable algorithm. From a methodological point of view, the approach in this work is different from previous ones in the literature, where normally the entangled state is first divided into apparently inequivalent coarse-grained sets under certain criteria (like range [21], partition number [8], ranks [22], etc.) and then more fine-grained classification procedures are performed. This new approach might also be useful in the study of classification under SLOCC or other characters of the general multipartite entangled state. Finally, it should be mentioned that the tensor decomposition method may also play an important role in other entanglement property studies [23].

\section{Acknowledgments}

This work was supported in part by the National Natural Science Foundation of China(NSFC), by the CAS Key Projects KJCX2-yw-N29 and H92A0200S2. 


\section{References}

[1] M.A. Nielsen and I.L. Chuang, Quantum Computation and Quantum Information (Cambridge University Press, Cambridge, England, 2000).

[2] W. Dür, G. Vidal and J.I. Cirac, Phys. Rev. A 62, 062314 (2000).

[3] H.A. Carteret, A. Higuchi and A. Sudbery, J. Math. Phys. 41, 7932 (2000).

[4] Frank Verstraete, Jeroen Dehaene, and Bart De Moor, Phys. Rev. A 68, 012103 (2003).

[5] B. Kraus, Phys. Rev. Lett. 104, 020504 (2010).

[6] Ryszard Horodecki, Paweł Horodecki, Michał Horodecki and Karol Horodecki, Rev. Mod. Phys. 81, 865 (2009).

[7] Tsubasa Ichikawa, Toshihiko Sasaki, Izumi Tsutsui, and Nobuhiro Yonezawa, Phys. Rev. A 78, 052105 (2008).

[8] T. Bastin, S. Krins, P. Mathonet, M. Godefroid, L. Lamata and E. Solano, Phys. Rev. Lett. 103, 070503 (2009).

[9] Damian.J.H. Markham, Phys. Rev. A 83, 042332 (2011).

[10] P. Ribeiro and R. Mosseri, Phys. Rev. Lett. 106, 180502 (2011).

[11] Martin Aulbach, arXiv:1103.0271.

[12] Xikun Li, Junli Li, Bin Liu and Cong-Feng Qiao, Sci. China G 54, 1471 (2011).

[13] David W. Lyons and Scott N. Walck, Phys. Rev. A 78, 042314 (2008).

[14] Curt D. Cenci, David W. Lyons, Laura M. Snyder and Scott N. Walck, Quantum. Inform. Compu. 10, 1029 (2010).

[15] Curt D. Cenci, David W. Lyons and Scott N. Walck, arXiv:1011.5229.

[16] L.D. Lathauwer, B.D. Moor and J. Vandewalle, SIAM J. Matrix Anal. Appl. 21, 1253 $(2000)$. 
[17] Tamara G. Kolda and Brett W. Bader, SIAM Review 51, 455 (2009).

[18] Aviad Kipnis and Adi Shamir, Lect. Notes Comput. Sci. 1666, 19 (1999).

[19] Nicolas Courtois, Alexander Klimov, Jacques Patarin, and Adi Shamir, Lect. Notes Comput. Sci. 1807, 392 (2000).

[20] Jun-Li Li and Cong-Feng Qiao, arXiv:1111.4379

[21] Lin Chen and Yi-Xin Chen, Phys. Rev. A 73, 052310 (2006).

[22] Shuo Cheng, Junli Li and Cong-Feng Qiao, J. Phys. A 43, 055303 (2010).

[23] Joseph J. Hilling and Anthony Sudbery, J. Math. Phys. 51, 072102 (2010). 